\title{
Statistics of coherent waves inside media with Lévy disorder
}

\author{
Luis A. Razo-López $\odot,{ }^{1}$ Azriel Z. Genack $\odot,{ }^{2,3}$ and Victor A. Gopar $\oplus^{4, *}$ \\ ${ }^{1}$ Institut de Physique de Nice, Universitè Côte d'Azur, CNRS, Parc Valrose, 06100 Nice, France \\ ${ }^{2}$ Department of Physics, Queens College of the City University of New York, Flushing, New York 11367, USA \\ ${ }^{3}$ The Graduate Center of the City University of New York, New York, New York 10016 USA \\ ${ }^{4}$ Departamento de Física Teórica, Facultad de Ciencias, and BIFI, Universidad de Zaragoza, Pedro Cerbuna 12, 50009 Zaragoza, Spain
}

(Received 31 December 2020; accepted 9 February 2021; published 9 April 2021)

\begin{abstract}
Structures with heavy-tailed distributions of disorder occur widely in nature. The evolution of such systems, as in foraging for food or the occurrence of earthquakes, is generally analyzed in terms of an incoherent series of events. But the study of wave propagation or lasing in such systems requires the consideration of coherent scattering. We consider the distribution of wave energy inside one-dimensional random media in which the spacing between scatterers follow a Lévy $\alpha$-stable distribution characterized by a power-law decay with exponent $\alpha$. We show that the averages of the intensity and logarithmic intensity are given in terms of the average of the logarithm of transmission and the depth into the sample raised to the power $\alpha$. Mapping the depth into the sample to the number of scattering elements yields intensity statistics that are identical to those found for Anderson localization in standard random media. This allows for the separation for the impacts of disorder distribution and wave coherence in random media.
\end{abstract}

DOI: 10.1103/PhysRevResearch.3.023035

\section{INTRODUCTION}

The average motion of particles in space and time in samples in which at least the first two moments of the distribution of spacing are finite follows a diffusion equation in Brownian models. Coherent-transport phenomena of classical and quantum waves have been also studied using Brownian approaches $[1,2]$. Diffusion is suppressed as a result of coherent backscattering in which waves returning to points in the medium along time-reversed paths interfere constructively. Anderson localization occurs as diffusion ceases in sufficiently large systems of dimensions $d \leqslant 2$ and in higher dimensions above a critical value of disorder [3-6]. In one dimension, all waves are localized. The average transmission falls exponentially asymptotically, $\langle T\rangle \sim \exp (-L / \ell)$, while $\langle\ln T\rangle=-L / \ell$. Indeed, the full statistics of transmission in standard light-tailed distributions of separation between scattering elements is determined in accordance with the single-parameter scaling theory of localization in terms of the dimensionless parameter $L / \ell[2,7]$.

Most studies of coherent transport in random media consider standard light-tailed distributions of disorder that lead to Anderson localization. Such systems include mesoscopic electronic systems in micron-scale devices at low temperatures and classical waves in stationary media. However,

\footnotetext{
*gopar@unizar.es

Published by the American Physical Society under the terms of the Creative Commons Attribution 4.0 International license. Further distribution of this work must maintain attribution to the author(s) and the published article's title, journal citation, and DOI.
}

heavy-tailed distributions are common in biology and geology [8] and may lead to advantageous mesoscopic devices.

Heavy-tailed Lévy $\alpha$-stable distribution are characterized by power-law tails. Thus, for a random variable $z$ following a Lévy $\alpha$-stable distribution $\rho(z)$ [9-11],

$$
\rho(z) \sim 1 / z^{1+\alpha}
$$

for $z \gg 1$ and $0<\alpha<2$. For $\alpha<1$, both the first and second moments diverge.

In Lévy-type disorder, waves can travel long distances without being scattered and thus have a profound impact on the transport properties. Measurements and analytic calculations of wave transmission in Lévy $\alpha$-stable media give different scaling than in standard one-dimensional (1D) random media $[8,12-26]$.

In this work, we treat the energy inside heavy-tailed Lévy disordered media. The distinctive impacts of Lévy $\alpha$-stable disorder and Anderson localization upon wave propagation are manifest. Potential applications of novel states in Lévy disordered media for low-threshold lasing are discussed.

In particular, we investigate the statistics of waves inside a 1D Lévy disordered structures via the statistics of intensity $I(x)$ at the observation point $x$ (see Fig. 1).

As we show below, for Lévy-type disorder, the average of the intensity and its logarithm follow power-law dependences with the observation point. We will contrast these results with those corresponding to systems with standard disorder, which has been studied experimentally and theoretically [27-29]. Calculations of intensity inside Lévy disordered samples [30] using the concept of leap-over to compute the density of scatterers give different results from those presented below in Fig. 4 (solid lines). 


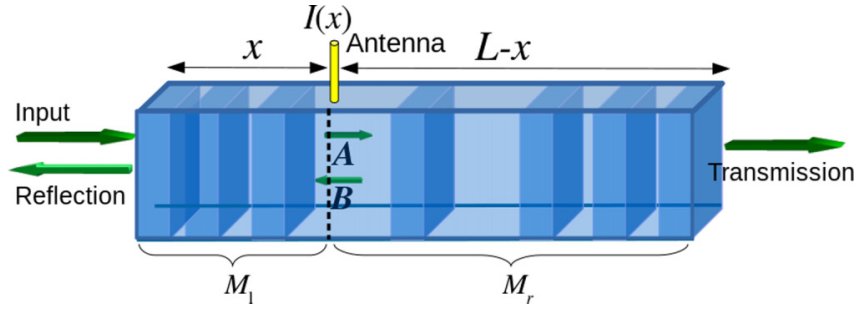

FIG. 1. Schematic of a random waveguide with scatterers (slabs) randomly separated according to a Lévy distribution. The scattering processes to the left and right of the observation point $x$ are described by the transfer matrices $M_{l}$ and $M_{r}$, respectively.

This paper is organized as follows. In Sec. II, we present general expressions for the intensity and transmission in a single sample in terms of transfer matrices. We then introduce some known results for the statistics of the transmission of standard disordered systems that will be contrasted with the statistics of systems with Lévy disorder in the subsequent section. In Sec. III, Lévy-type disorder is introduced and compared to transmission in standard systems. The results for the transmission are useful in the study of the statistics of the intensity inside the medium. The averages of the intensity and of the logarithm of intensity are given as functions of depth in the sample. Examples of the complete distribution of the logarithmic intensity are shown in Sec. III. A summary of the results and discussion are given in Sec. IV.

\section{PRELIMINARIES}

\section{A. Transfer matrix: Transmission and intensity}

Let us assume that we measure the intensity $I(x)$ at a point $x$. If $A$ and $B$ are the amplitudes of the forward and backward going waves at this point (see Fig. 1), $I(x)$ is given by

$$
I(x)=|A \exp (i k x)+B \exp (-i k x)|^{2},
$$

where $k$ is the wave number. We now introduce the transfer matrices $M_{l}$ and $M_{r}$ associated with the segments of the sample on the left- and right-hand sides of the observation point, respectively:

$$
M_{l(r)}=\left[\begin{array}{ll}
\gamma_{l(r)} & \beta_{l(r)} \\
\beta_{l(r)}^{*} & \gamma_{l(r)}^{*}
\end{array}\right]
$$

where $\gamma_{l(r)}$ and $\beta_{l(r)}$ are complex numbers satisfying $\left|\gamma_{l(r)}\right|^{2}-$ $\left|\beta_{l(r)}\right|^{2}=1$. The amplitudes $A$ and $B$ can be written in terms of the transfer matrices $M_{l(r)}$, and from Eq. (2), the intensity is given by

$$
\begin{aligned}
I(x) & =\frac{1}{\left|\gamma_{r}\right|^{2}}\left|\gamma_{l} \gamma_{r}^{*}+\beta_{l} \beta_{r}^{*}\right|^{2}\left|1-\frac{\beta_{r}^{*}}{\gamma_{r}^{*}} \exp -2 i k x\right|^{2} \\
& =\frac{T}{T_{r}}\left|1-\frac{\beta_{r}^{*}}{\gamma_{r}^{*}} \exp (-2 i k x)\right|^{2},
\end{aligned}
$$

where $T=\left|\gamma_{l} \gamma_{r}^{*}+\beta_{l} \beta_{r}^{*}\right|^{2}$ is the transmission coefficient of the entire sample and $T_{r}=1 /\left|\gamma_{r}\right|^{2}$ is the transmission coefficient of the right segment.
The transfer matrices $M_{l(r)}$ are conveniently written in the polar representation as [2]

$$
M_{l(r)}=\left[\begin{array}{cc}
\sqrt{1+\lambda_{l(r)}} e^{i \theta_{l(r)}} & \sqrt{\lambda_{l(r)}} e^{i\left(2 \mu_{l(r)}-\theta_{l(r)}\right)} \\
\sqrt{\lambda_{l(r)}} e^{-i\left(2 \mu_{l(r)}-\theta_{l(r)}\right)} & \sqrt{1+\lambda_{l(r)}} e^{-i \theta_{l(r)}}
\end{array}\right],
$$

with phases $\theta_{l(r)}, \mu_{l(r)} \in[0,2 \pi]$ and $\lambda_{l(r)} \geqslant 0$. An advantage of using the polar representation is that the radial variables $\lambda_{l(r)}$ are directly related to the transmission coefficients: $\lambda_{l(r)}=\left(1-T_{l(r)}\right) / T_{l(r)}$. Therefore, Eq. (4) can be written as

$$
I(x)=\frac{T}{T_{r}}\left|1-\sqrt{1-T_{r}} \exp \left[-2 i\left(\mu_{r}-\theta_{r}+k x\right)\right]\right|^{2},
$$

while the total transmission $T$ is given by

$$
\begin{aligned}
\frac{1}{T}= & \frac{1}{T_{r} T_{l}}\left[2+T_{r} T_{l}-T_{r}-T_{l}\right. \\
& \left.+2 \sqrt{\left(T_{r}-1\right)\left(T_{l}-1\right) \cos 2\left(\mu_{l}-\mu_{r}+\theta_{r}\right)}\right] .
\end{aligned}
$$

\section{B. Statistics of the transmission in standard disordered 1D media}

Now that we have obtained analytical expressions for the intensity and transmission of a single sample in the previous section, we consider an ensemble of random samples. In particular, we assume that the disordered structures are composed of randomly separated weak scatterers or slabs. Thus, the intensity $I(x)$ is a random quantity. From Eq. (5), the statistics of $I(x)$ depend on the statistical properties of the transmission and the angular variables $\theta_{l(r)}$ and $\mu_{l(r)}$.

Before considering the case of disordered samples with Lévy disorder, we introduce the distribution of the transmission for standard disorder. The statistics of transmission through standard disordered systems with light-tailed distributions have been extensively studied using random matrix theory $[1,2]$. The distribution of the transmission $p_{s}(T)$ is given by $[31,32]$

$$
p_{s}(T)=C \frac{[\operatorname{acosh}(1 / \sqrt{T})]^{1 / 2}}{T^{3 / 2}(1-T)^{1 / 4}} e^{-s^{-1} \operatorname{acosh}^{2}(1 / \sqrt{T})},
$$

where $C$ is a normalization constant and $s=L / \ell$, with $L$ being the length of the system and $\ell$ being the mean free path. The complete distribution of transmission is determined by the parameter $s$, which is proportional to the number of scatterers $n$ in the sample with proportionality constant $a: s=a n$ [33].

For later comparisons with systems with Lévy disorder, we point out the asymptotic exponential decay with $L$ of the average transmission in standard disordered systems [1]:

$$
\langle T\rangle \propto \exp (-L / 2 \ell),
$$

and the linear behavior of the average of the logarithmic transmission

$$
\langle-\ln T\rangle \propto L .
$$

To illustrate some statistical properties of the transmission of standard disordered systems, we show in Fig. 2(a) the distribution of the logarithmic transmission $p_{s}(\ln T)$, which is obtained from Eq. (7), and the linear behavior of $\langle-\ln T\rangle$ with $L$, given by Eq. (9). The histogram and symbols in Fig. 2 are obtained from numerical simulations, as explained below. 


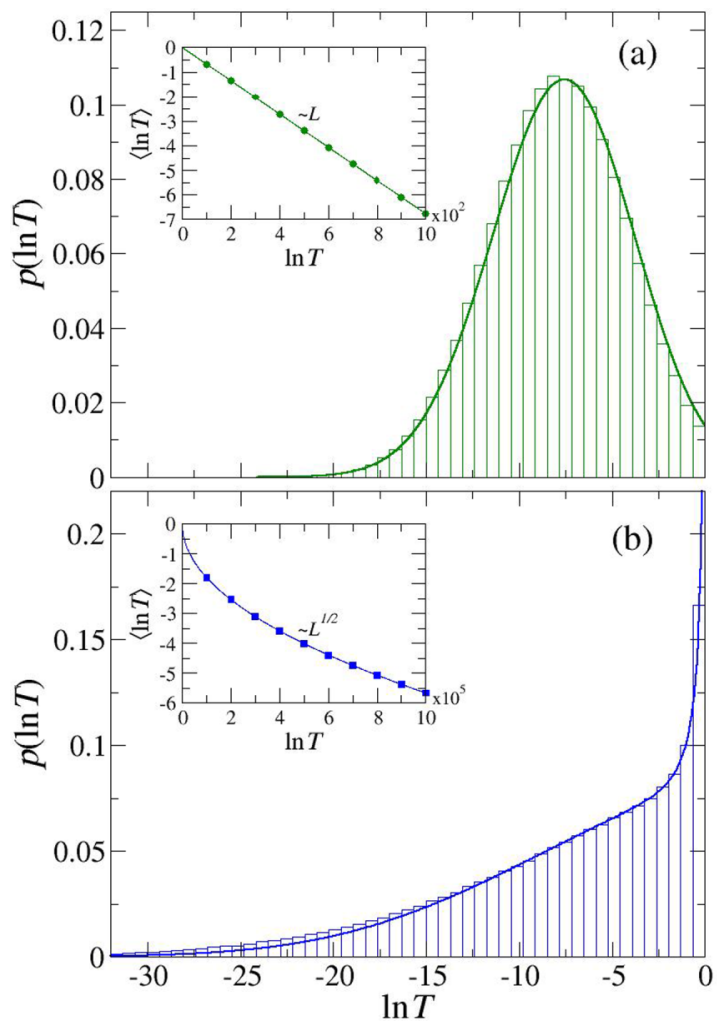

FIG. 2. (a) The distribution of the logarithmic transmission for standard disorder systems. The distribution is determined by the parameter $s=\langle-\ln T\rangle=8$. Inset: linear behavior of $\langle\ln T\rangle$ with the system length. (b) The distribution of the logarithmic transmission for Lévy disordered systems. The distribution is determined by the parameters $\langle-\ln T\rangle=8$ and $\alpha=1 / 2$. Inset: the power-law behavior of $\langle\ln T\rangle$ with the system length, Eq. (12).

The numerical simulations performed in this work are based on the transfer matrix approach $[18,34]$. The numerical model consists of layers $2.5 \mathrm{~mm}$ thick with refraction index $n_{2}=1.1$, randomly placed in a background with index of refraction $n_{1}=1$ with separations following a Gaussian distribution for standard disorder and a Lévy $\alpha$-stable distribution for Lévy disorder. We have fixed the frequency at $1 \mathrm{THz}$ in all the calculations. At this frequency, the layers have a reflection coefficient of 0.007 . Statistics are collected for $10^{6}$ realizations of the disorder.

\section{STATISTICS OF THE INTENSITY INSIDE 1D MEDIA WITH LÉVY DISORDER}

We utilize the model of Lévy disordered media introduced in [15] with the asymptotic decay given in Eq. (1). We briefly summarize the main results of Ref. [15] for the transmission that will be useful for obtaining the statistical properties of the intensity. We will consider the case $\alpha<1$, where the effects of Lévy disorder on transport are strong.

In Lévy disordered samples of fixed length $L$, the number of scattering units $n$ is a random variable with strong sample-to-sample fluctuations; thus, it is crucial to know the complete distribution of $n$. The probability density $\Pi_{L}(n ; \alpha)$ of these fluctuations is given by [15]

$$
\Pi_{L}(n ; \alpha)=\frac{2}{\alpha} \frac{L}{(2 n)^{\frac{1+\alpha}{\alpha}}} q_{\alpha, c}\left[L /(2 n)^{1 / \alpha}\right]
$$

for $0<\alpha<1$ in the limit $L \gg c^{1 / \alpha}$, with $c$ being a scaling parameter. The probability density $q_{\alpha, c}(x)$ has a power-law tail: $q_{\alpha, c}(x) \sim c / x^{1+\alpha}$ for $x \gg 1$.

Using the distribution of the transmission for a fixed number of scatterers $p_{s}(T)$ given in Eq. (7) and the distribution $\Pi_{L}(n, \alpha)$, Eq. (10), we write the distribution of the transmission for Lévy disordered systems $p_{\alpha, \xi}(T)$ as

$$
P_{\alpha, \xi}(T)=\int_{0}^{\infty} p_{s(\alpha, \xi, z)}(T) q_{\alpha, 1}(z) d z
$$

where $p_{s(\alpha, \xi, z)}(T)$ is given by Eq. (7), with $s$ replaced by $s(\alpha, \xi, z)=\xi /\left(2 z^{\alpha} \mathcal{I}_{\alpha}\right)$ and $\mathcal{I}_{\alpha}$ equal to half of the mean value $\left\langle z^{-\alpha}\right\rangle: \mathcal{I}_{\alpha}=(1 / 2) \int z^{-\alpha} q_{\alpha, 1} d z=\cos (\pi \alpha / 2) / 2 \Gamma(1+$ $\alpha)$, where $\Gamma$ denotes the gamma function [35]. The parameter $\xi$ introduced in Eq. (11) is the average of the logarithmic transmission for a fixed length $L$ : $\xi=\langle-\ln T\rangle_{L}=$ $\int_{0}^{\infty} a n \Pi_{L}(n) d n$, which is given by

$$
\langle\ln T\rangle_{L}=-\left(\frac{a}{c} \mathcal{I}_{\alpha}\right) L^{\alpha}
$$

Since the factor in parentheses in Eq. (12) is a constant, the average of the logarithmic transmission is a power-law function of $L$, in contrast to the linear dependence for standard disorder in Eq. (9). Similarly, a power law is found for the average transmission [18] $\langle T\rangle \propto L^{-\alpha}$ in contrast to the exponential decay for standard disordered systems in Eq. (8).

In Fig. 2(b), we show an example of the distribution of the logarithm of transmission for Lévy disordered structures characterized by $\alpha=1 / 2$. The theoretical results as given in Eq. (11) are compared to numerical simulations shown by the histogram. The power-law behavior of $\langle\ln T\rangle$ in Lévy structures is shown in the inset in Fig. 2(b). Thus, by comparing Figs. 2(a) and 2(b), the strong influence of Lévy disorder on the statistical properties of transmission is clearly seen. We also note that the only parameters that enter into Eq. (11) are $\alpha$ and $\xi=\langle\ln T\rangle$. Thus, the complete statistics of transmission is determined by these parameters.

With the above results for the statistics of transmission, we now study the statistical properties of the intensity. As we show next, the presence of Lévy disorder is revealed in basic statistical quantities such as the ensemble averages $\langle\ln I(x)\rangle_{L}$ and $\langle I(x)\rangle_{L}$.

Let us consider first the average of the logarithmic intensity $\langle\ln I(x)\rangle$. The calculations are lengthy, but a simple analytical result is obtained. $\langle\ln I(x)\rangle$ is of particular importance since it is directly related to the average of the logarithmic transmission, which along $\alpha$ determines all the statistical properties of the transport in Lévy disordered systems.

We perform the average over the uniformly distributed phases in Eq. (5) to obtain $\langle\ln I(x)\rangle=\langle\ln T\rangle_{L}-\left\langle\ln T_{r}\right\rangle_{L-x}$. Since $\ln T$ is an additive quantity, we obtain $\langle\ln T\rangle_{L-x}=$ 

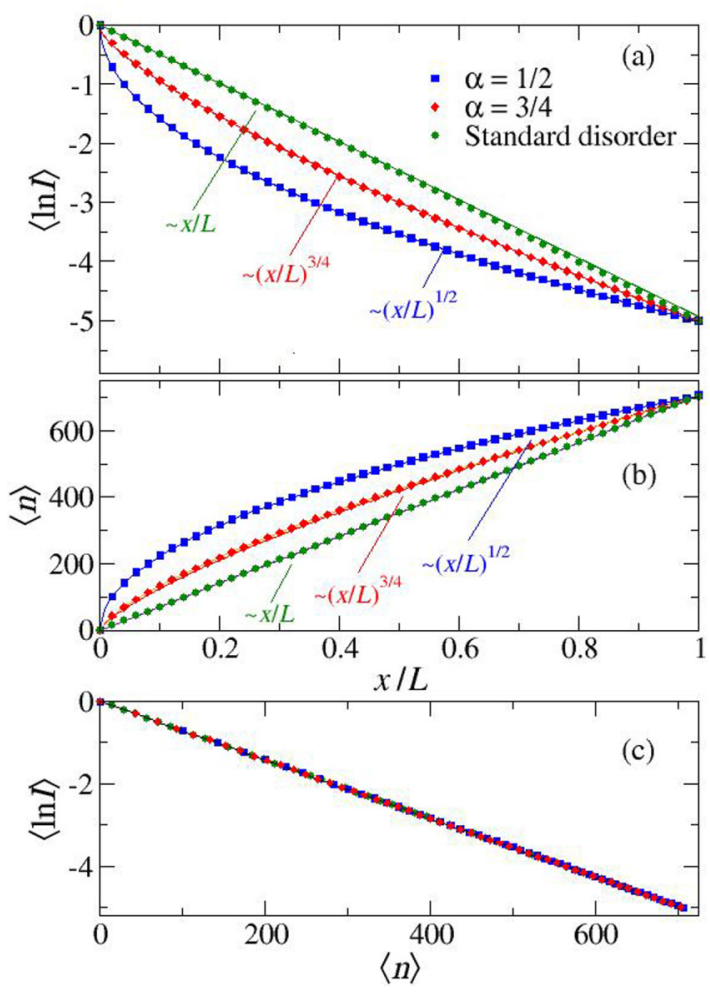

FIG. 3. (a) Average of the logarithmic intensity for Lévy disordered systems with $\alpha=1 / 2$ and 3/4 (red and blue symbols). The solid lines are given by Eq. (13). For comparison, the linear dependence on $x$ in the case of standard disorder is also shown (green). In all cases, $\langle-\ln T\rangle_{L}=5$. (b) The average number of slabs $\langle n\rangle$ in the Lévy $(\alpha=1 / 2,3 / 4)$ and standard disordered structures at the position of observation $x$. (c) $\langle\ln I\rangle$ as a function of $\langle n\rangle$ for $\alpha=1 / 2,3 / 4$ and standard disorder.

$\langle\ln T\rangle_{L}-\langle\ln T\rangle_{x}$, and from Eq. (10), we have

$$
\begin{aligned}
\langle\ln I(x)\rangle & =-\left(\frac{a}{c} \mathcal{I}_{\alpha}\right) x^{\alpha} \\
& =\langle\ln T\rangle_{L}\left(\frac{x}{L}\right)^{\alpha} .
\end{aligned}
$$

Thus, $\langle\ln I(x)\rangle \propto x^{\alpha}$ has a power-law behavior in Lévy disordered media in contrast to the linear dependence in standard disordered media [29].

We verify the result given in Eq. (13) numerically. The results (symbols) are shown in Fig. 3(a) together with the theoretical results (solid lines) from Eq. (13) for $\alpha=1 / 2$ and $3 / 4$. The linear behavior of $\langle\ln I(x)\rangle$ for standard disorder is also shown in Fig. 3(a) (green squares).

$\langle\ln I(x)\rangle$ does not fall linearly in Lévy structures as in standard structures [Fig. 3(a)]; however, the role of coherent backscattering in inhibiting propagation is unchanged. To gain insight into this nonlinear behavior, we note that $\langle\ln I(x)\rangle$ is given by the difference between the average of the logarithmic transmission of the complete sample $(L)$ and the right segment $(L-x)$, as we have shown above. The power-law behavior of $\langle\ln I(x)\rangle$ finds its origin in the power law of the variation of the number of scatterers up to the depth $x$. This is illustrated in Fig. 3(b), where the average number of scatterers $\langle n\rangle=$

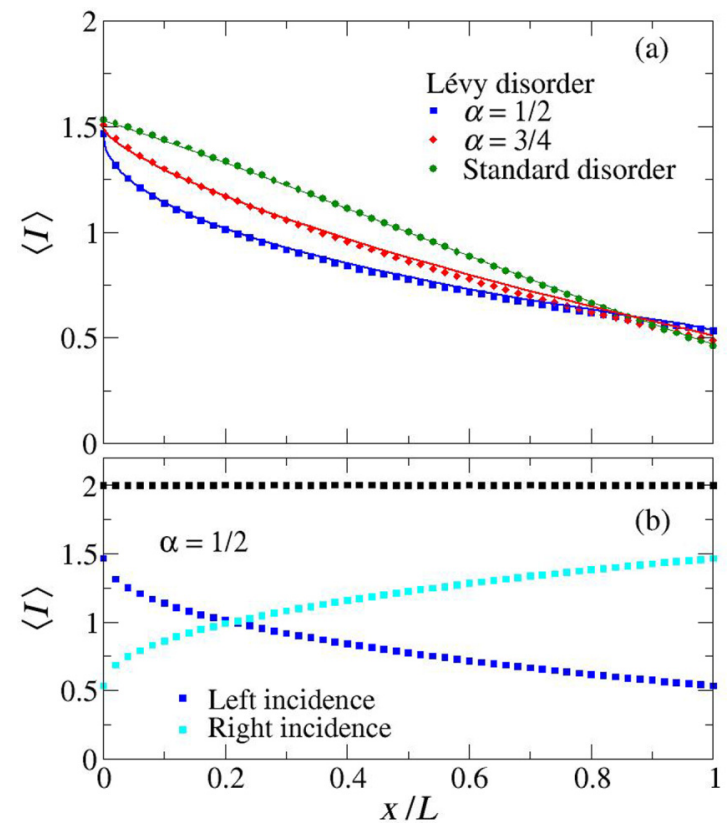

FIG. 4. (a) Ensemble average intensity $\langle I\rangle$ for Lévy $\alpha=1 / 2$ and $3 / 4$ and standard disorder. The solid lines are obtained from Eq. (14). In all three cases $\langle-\ln T\rangle=1$. (b) The average $\langle I\rangle$ for $\alpha=1 / 2$, as in (a), but for waves incident from the left and right incidences. The black squares show that the sum of both incidences is constant.

$\int n \Pi_{x}(n) d n=\mathcal{I}_{\alpha} x^{\alpha} / c$ is plotted at the position $x / L$ for Lévy $(\alpha=1 / 2,3 / 4)$ and standard disordered structures. For Lévy disordered samples, the average number of scatterers follows a power law with exponent $\alpha$. In contrast, in standard disorder, the average number of scatterers is a linear function of system size. Thus, for both Lévy and standard disorders, $\langle\ln I\rangle$ is a linear function of $\langle n\rangle$, as shown in Fig. 3(c), and $\langle\ln I(x)\rangle$ is additive, as in standard disordered structures [29,36].

We now study the ensemble average intensity $\langle I(x)\rangle$; after averaging $I(x)$, Eq. (4), over the uniformly distributed random phases, we write $\langle I(x)\rangle$ as [28]

$$
\langle I(x)\rangle=\int_{0}^{1} \int_{0}^{1} \frac{T_{l}\left(2-T_{r}\right)}{T_{l}+T_{r}-T_{l} T_{r}} P_{\alpha, \xi_{l}}\left(T_{l}\right) P_{\alpha, \xi_{r}}\left(T_{r}\right) d T_{l} d T_{r},
$$

where the distributions $P_{\alpha, \xi_{l}}\left(T_{l}\right)$ and $P_{\alpha, \xi_{r}}\left(T_{r}\right)$ are the distributions for the left and right segments, respectively, with $\xi_{l}=\left\langle\ln T_{l}\right\rangle=(x / L)^{\alpha}\langle\ln T\rangle_{L}$ and $\xi_{r}=\left\langle\ln T_{r}\right\rangle=$ $\left(1-(x / L)^{\alpha}\right)\langle\ln T\rangle_{L}$, according to Eq. (12). We can verify the particular cases at $x=0$ and $x=L$ : for $x=0, T_{l}=1$ and $\langle I(0)\rangle=\left\langle\left(2-T_{r}\right)\right\rangle=2-\langle T\rangle$, while at $x=L, T_{r}=1$ and therefore $\langle I(L)\rangle=\left\langle T_{r}\right\rangle=\langle T\rangle$.

We perform numerical simulations to support Eq. (14), in which the double integral is performed numerically. The distribution for the left segment $P_{\alpha, \xi_{l}}\left(T_{l}\right)$ in Eq. (14) is obtained from Eq. (11), while for the right segment, $P_{\alpha, \xi_{r}}\left(T_{r}\right)$ is obtained by considering the corresponding probability density of scatterers which is generated numerically. The results are shown in Fig. 4(a) for $\alpha=1 / 2$ and 3/4. The numerical simulations and Eq. (14) are seen to be in good agreement. The average intensity for standard disorder is also shown (green 


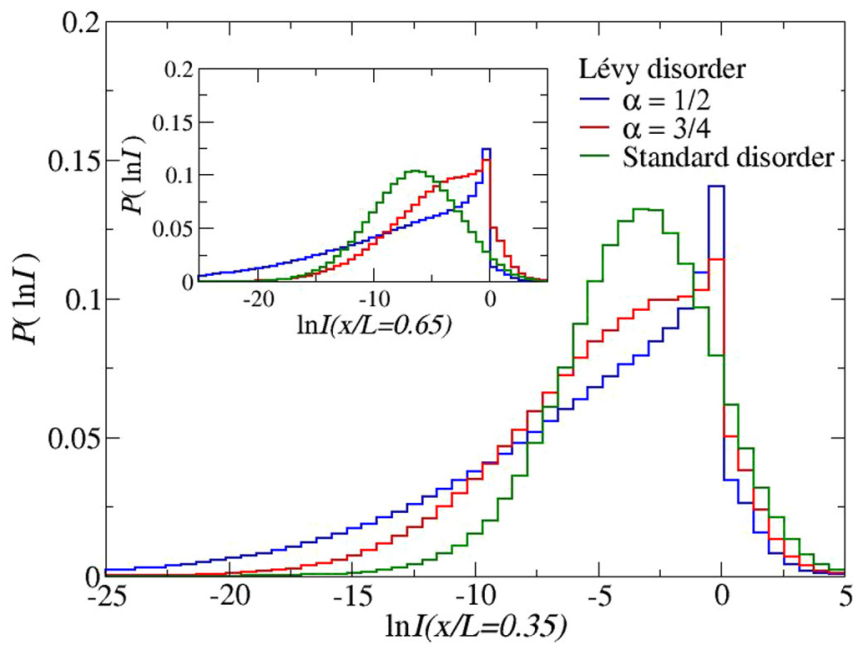

FIG. 5. Distribution of the logarithmic intensity for Lévy and standard disordered structures. Here $\langle-\ln T\rangle=10$.

dots and solid line) in Fig. 4(a) to provide a contrast with the power-law dependence found in media with Lévy disorder.

The profile of $\langle I(x)\rangle$ is not symmetric about the center, as it is in standard homogeneously disordered systems [see Fig. 4(a)]. In Lévy disordered structures, the disorder is inhomogeneous; the density of scatterers is greatest near the left side of the sample, as can be seen in Fig. 3(b), where waves launched, causing the intensity to fall more rapidly there. However, the sum of intensities for waves incident from the left and right is constant and equal to twice the intensity of the incident beam from one side, as shown in Fig. 4(b). This can be understood by noting that for the integrand of Eq. (14) for the wave incident from the right, $T_{l}$ and $\xi_{l}$ are replaced by $T_{r}$ and $\xi_{r}$, respectively, and $T_{r}$ and $\xi_{r}$ are replaced by $T_{l}$ and $\xi_{l}$. Adding the contributions from the waves incident from the left and right gives $T_{l}\left(2-T_{r}\right) /\left(T_{l}+T_{r}-T_{l} T_{r}\right)+T_{r}(2-$ $\left.T_{l}\right) /\left(T_{l}+T_{r}-T_{l} T_{r}\right)=2$. Since $P_{\alpha, \xi_{l,(r)}}\left(T_{l,(r)}\right)$ are normalized, the average of the sum of intensities excited by waves incident on the left and right is equal to 2 . This result is illustrated in Fig. 4(b) for the case of $\alpha=1 / 2$, where the black squares are the sum of the average intensity for incident waves from the left and right ends of the samples.

The previous discussion is general and gives an average intensity profile for standard homogeneously disordered media which is symmetric about the center of the sample [36]. This symmetry is summarized by the expression $\langle I(x)\rangle+\langle I(L-$ $x)\rangle=2$ and reflects the fact that the sum of intensities from the right and left is equal to the local density of states (LDOS) relative to LDOS outside the medium, which is unchanged by disorder.

The complete distribution of the logarithm is obtained numerically and shown in Fig. 5 for $\langle\ln T\rangle=10$. There is a higher probability of large fluctuations of intensity in Lévy disordered samples (blue and red histograms) compared to standard disordered systems (green histogram).

\section{SUMMARY AND DISCUSSION}

We have studied the wave intensity statistics inside random 1D media with disorder described by Lévy-type distributions characterized by an asymptotic power-law decay. For both $\langle I(x)\rangle$ and $\langle\ln I(x)\rangle$, we find a power-law decay with position. In contrast, $\langle I(x)\rangle$ falls linearly in standard disordered systems. The slower decay with $x$ than for the standard disorder indicates that wave localization in space is weaker in Lévy disorder than in standard disorder.

The equivalence of the statistics of intensity in $\alpha$-stable Lévy disordered systems and standard random media at the corresponding layer number $n$ suggests opportunities for engineering structures for analyzing and controlling waves. The forward and backward amplitudes within a layer of the medium are constant so that they interfere and create an oscillatory pattern with high peak intensity within the layer. When a thick layer is near the spatial and spectral peak of a mode, the lifetime of the quasinormal mode increases, and the line narrows as the thickness of the layer increases. The material could therefore serve as a filter. If gain is introduced into this system, the correspondingly long lifetime of the mode would enhance the opportunity for emitted photons to stimulate emission before escaping the sample [37]. In addition, the large spatial extent of the mode allows the system to be efficiently pumped without saturating the gain medium. The prospects for an $\alpha$-stable laser will be considered in future work.

\section{ACKNOWLEDGMENTS}

We acknowledge discussions with $\mathrm{X}$. Ma. This work is supported by the National Science Foundation under EAGER Award No. 2022629, by PSC-CUNY under Award No. 63822-00 51, and by MCIU (Spain) under Project No. PGC2018-094684-B-C22. L.A.R.-L. thanks UCA ${ }^{\mathrm{JEDI}}$ for the award of a predoctoral studies fellowship.
[1] C. W. J. Beenakker, Random-matrix theory of quantum transport, Rev. Mod. Phys. 69, 731 (1997).

[2] P. Mello and N. Kumar, Quantum Transport in Mesoscopic Systems: Complexity and Statistical Fluctuation (Oxford University Press, Oxford, 2004).

[3] P. W. Anderson, Absence of diffusion in certain random lattices, Phys. Rev. 109, 1492 (1958).

[4] E. Abrahams, P. W. Anderson, D. C. Licciardello, and T. V. Ramakrishnan, Scaling Theory of Localization: Absence of
Quantum Diffusion in Two Dimensions, Phys. Rev. Lett. 42, 673 (1979).

[5] E. Abrahams, 50 Years of Anderson Localization (World Scientific, Singapore, 2010).

[6] B. Kramer and A. MacKinnon, Localization: Theory and experiment, Rep. Prog. Phys. 56, 1469 (1993).

[7] P. W. Anderson, D. J. Thouless, E. Abrahams, and D. S. Fisher, New method for a scaling theory of localization, Phys. Rev. B 22, 3519 (1980). 
[8] V. Zaburdaev, S. Denisov, and J. Klafter, Lévy walks, Rev. Mod. Phys. 87, 483 (2015).

[9] P. Lévy, Theory of Summation of Random Variables (GauthiersVillars, Paris, 1937).

[10] B. V. Gnedenko and A. N. Kolmogorov, Limit distributions for sums of independent random variables, Math. Gaz. 39, 342 (1955).

[11] V. V. Uchaikin and V. M. Zolotarev, Chance and Stability: Stable Distributions and Their Applications (Wiley, New York, 1998).

[12] M. Leadbeater, V. I. Falko, and C. J. Lambert, Lévy Flights in Quantum Transport in Quasiballistic Wires, Phys. Rev. Lett. 81, 1274 (1998).

[13] P. Barthelemy, J. Bertolotti, and D. S. Wiersma, A Lévy flight for light, Nature (London) 453, 495 (2008).

[14] N. Mercadier, W. Guerin, M. Chevrollier, and R. Kaiser, Lévy flights of photons in hot atomic vapours, Nat. Phys. 5, 602 (2009).

[15] F. Falceto and V. A. Gopar, Conductance through quantum wires with Lévy-type disorder: Universal statistics in anomalous quantum transport, Europhys. Lett. 92, 57014 (2010).

[16] I. Amanatidis, I. Kleftogiannis, F. Falceto, and V. A. Gopar, Conductance of one-dimensional quantum wires with anomalous electron wave-function localization, Phys. Rev. B 85, 235450 (2012).

[17] A. A. Fernández-Marín, J. A. Méndez-Bermúdez, J. Carbonell, F. Cervera, J. Sánchez-Dehesa, and V. A. Gopar, Beyond Anderson Localization in 1D: Anomalous Localization of Microwaves in Random Waveguides, Phys. Rev. Lett. 113, 233901 (2014).

[18] A. A. Fernández-Marín, J. A. Méndez-Bermúdez, and V. A. Gopar, Photonic heterostructures with Lévy-type disorder: Statistics of coherent transmission, Phys. Rev. A 85, 035803 (2012).

[19] A. A. Asatryan and A. Novikov, Anderson localization of classical waves in weakly scattering one-dimensional Levy lattices, Phys. Rev. B 98, 235144 (2018).

[20] J. R. F. Lima, L. F. C. Pereira, and A. L. R. Barbosa, Dirac wave transmission in Lévy-disordered systems, Phys. Rev. E 99, 032118 (2019).

[21] L. A. Razo-López, A. A. Fernández-Marín, J. A. MéndezBermúdez, J. Sánchez-Dehesa, and V. A. Gopar, Delay time of waves performing Lévy walks in 1D random media, Sci. Rep. 10, 20816 (2020).

[22] D. Boosé and J. M. Luck, Statistics of quantum transmission in one dimension with broad disorder, J. Phys. A 40, 14045 (2007).
[23] C. W. J. Beenakker, C. W. Groth, and A. R. Akhmerov, Nonalgebraic length dependence of transmission through a chain of barriers with a Lévy spacing distribution, Phys. Rev. B 79, 024204 (2009).

[24] R. Burioni, L. Caniparoli, and A. Vezzani, Lévy walks and scaling in quenched disordered media, Phys. Rev. E 81, 060101(R) (2010).

[25] R. T. Sibatov, Distribution of the conductance of a linear chain of tunnel barriers with fractal disorder, JETP Lett. 93, 503 (2011).

[26] J.-P. Bouchaud and A. Georges, Anomalous diffusion in disordered media: Statistical mechanisms, models and physical applications, Phys. Rep. 195, 127 (1990).

[27] A. G. Yamilov, R. Sarma, B. Redding, B. Payne, H. Noh, and H. Cao, Position-Dependent Diffusion of Light in Disordered Waveguides, Phys. Rev. Lett. 112, 023904 (2014).

[28] P. A. Mello, Z. Shi, and A. Z. Genack, Reprint of: Connection between wave transport through disordered $1 \mathrm{D}$ waveguides and energy density inside the sample: A maximum-entropy approach, Phys. E (Amsterdam, Neth.) 82, 261 (2016).

[29] X. Cheng, X. Ma, M. Yépez, A. Z. Genack, and P. A. Mello, Single-parameter scaling and maximum entropy inside disordered one-dimensional systems: Theory and experiment, Phys. Rev. B 96, 180203(R) (2017).

[30] X. Ma and A. Z. Genack, Anomalous wave transport in onedimensional systems with Levy disorder, arXiv:1807.05788.

[31] V. A. Gopar and R. A. Molina, Controlling conductance statistics of quantum wires by driving ac fields, Phys. Rev. B 81, 195415 (2010).

[32] I. Kleftogiannis, I. Amanatidis, and V. A. Gopar, Conductance through disordered graphene nanoribbons: Standard and anomalous electron localization, Phys. Rev. B 88, 205414 (2013).

[33] P. A. Mello, Central-limit theorems on groups, J. Math. Phys. 27, 2876 (1986).

[34] P. Markos and C. M. Soukoulis, Wave Propagation: From Electrons to Photonic Crystals and Left-Handed Materials (Princeton University Press, Princeton, NJ, 2008).

[35] T. Koren, M. A. Lomholt, A. V. Chechkin, J. Klafter, and R. Metzler, Leapover Lengths and First Passage Time Statistics for Lévy Flights, Phys. Rev. Lett. 99, 160602 (2007).

[36] Y. Huang, C. Tian, V. A. Gopar, P. Fang, and A. Z. Genack, Invariance Principle for Wave Propagation inside Inhomogeneously Disordered Materials, Phys. Rev. Lett. 124, 057401 (2020).

[37] V. Milner and A. Z. Genack, Photon Localization Laser: LowThreshold Lasing in a Random Amplifying Layered Medium via Wave Localization, Phys. Rev. Lett. 94, 073901 (2005). 